\title{
Facial emotion and identity processing development in 5- to 15-year-old children
}

\author{
Patrick J. Johnston', Jordy Kaufman², Julie Bajic ${ }^{3}$, Alicia Sercombe 3 , Patricia T. Michie ${ }^{3}$ and Frini Karayanidis ${ }^{*}$ \\ ' Department of Psychology, University of York, York, UK \\ 2 Brain and Psychological Sciences Research Centre, Swinburne University of Technology, Melbourne, VIC, Australia \\ ${ }^{3}$ School of Psychology, University of Newcastle, Newcastle, NSW, Australia
}

\section{Edited by:}

Jessica Sommerville, University of

Washington, USA

\section{Reviewed by:}

Gustaf Gredebäck, Uppsala University, Sweden

Jessica Sommerville, University of Washington, USA

*Correspondence:

Frini Karayanidis, School of Psychology, University of Newcastle, Callaghan,

Newcastle, NSW 2308, Australia.

e-mail: frini.karayanidis@newcastle. edu.au
Most developmental studies of emotional face processing to date have focused on infants and very young children. Additionally, studies that examine emotional face processing in older children do not distinguish development in emotion and identity face processing from more generic age-related cognitive improvement. In this study, we developed a paradigm that measures processing of facial expression in comparison to facial identity and complex visual stimuli. The three matching tasks were developed (i.e., facial emotion matching, facial identity matching, and butterfly wing matching) to include stimuli of similar level of discriminability and to be equated for task difficulty in earlier samples of young adults. Ninety-two children aged 5-15 years and a new group of 24 young adults completed these three matching tasks. Young children were highly adept at the butterfly wing task relative to their performance on both face-related tasks. More importantly, in older children, development of facial emotion discrimination ability lagged behind that of facial identity discrimination.

Keywords: face processing, facial emotion, facial identity, development

\section{INTRODUCTION}

Does the manner in which faces are perceived and processed develop significantly through childhood? This is a fundamental question for developmental psychologists, neuroscientists as well as parents, and educators. While there is a certain level of mystery that will always surround how young infants see the faces of people around them, it is tempting to assume that older children see faces as adults do, since they clearly recognize their parents, siblings, teachers, and so on. Nonetheless, it is clear from research conducted over the past two decades that there is a great deal of development in how children recognize both identity and emotional expressions in the faces around them.

Since the rapid signaling capacity of the human face almost certainly predates the evolution of language, the ability to quickly, and accurately interpret the emotional or motivational state of other humans would intuitively appear to be a core component of social cognition. The ability to distinguish between particular individuals also has high value within the context of human social culture and is likely to represent a cognitive skill that is part of our early evolutionary legacy. Yet the ontogeny of this skill is less well understood. The overarching goal of this paper is to help determine whether this skill is available throughout childhood or continues to develop into adulthood. More specifically, the primary aim of this paper is to examine the relative developmental rate of facial identity and facial emotion processing abilities in young children and adolescents. There were three important motivators of this work. The first motivator was the scientific realization that there are distinct neural pathways and cognitive processes involved in the recognition of facial identity and facial affect (Bruce and Young, 1986; Haxby et al., 2000). The second motivator was the interesting prior work on children that generally shows age-related improvements in face processing tasks but is inconsistent with regard to whether this reflects improvements in face processing ability or general cognitive maturity. The third motivator was an understanding that much of the prior work with children has been subject to certain methodological confounds. This work represents our efforts to further clarify children's face processing through the minimization of these confounds.

Bruce and Young's (1986) seminal paper argued that the human ability to effortlessly glean important information from faces is subserved by distinct perceptual processes. These processes are said to include identity recognition and emotional expression recognition, as independently acting functional units that only share their outputs further upstream when higher cognitive processes come into play. Later electrophysiological and neuroimaging experiments have since affirmed that many face processing abilities are indeed accomplished through distinct and independent neural processes (e.g., Haxby et al., 2000).

The understanding that face processing is achieved through a set of abilities subserved by different mechanisms has inspired research with children to determine when and how these distinct mechanisms develop. Such research has now given us a much richer understanding of the visual worlds of infants and young children. For example, we know that 30-min-old infants can distinguish faces from non-faces (Johnson et al., 1991); and soon afterward recognize their mother's face (Bushnell et al., 1989; Pascalis et al., 1995). At approximately 5-6 months, infants are additionally capable of distinguishing different emotional expressions (de Haan and Nelson, 1997; WalkerAndrews, 1997; Leppänen and Nelson, 2006). Moreover, even at these young ages, it is evident that the distinct processes for different aspects of face processing are made possible by known differences in brain activity (de Haan and Nelson, 1997; Southgate et al., 2008). 
Notwithstanding the impressive abilities of infants, a great deal of development in face processing continues until much later in life. Studies examining emotion recognition in preschool children (e.g., Phillipot and Feldman, 1990; Boyatzis et al., 1993) show that emotion recognition accuracy improves with increasing age during this period. Children's ability to recognize different emotions emerges at different times, with happiness generally being recognized earlier than other emotions such as anger, fear, and surprise (Gross and Ballif, 1991; Smith and Walden, 1998). This is perhaps unsurprising since happy faces have been shown to occupy a more orthogonalized region of "face-space" than the other basic emotions (Johnston et al., 2001) and are therefore more easily discriminable. Indeed differences in accuracy to different emotion categories continue to be apparent through middle childhood (Herba et al., 2006; Gao and Maurer, 2009) and can be shown to occur in healthy adults even when ceiling effects are avoided (Johnston et al., 2003, 2006). In addition, typically, error patterns made by young children are qualitatively similar to those made by adults (i.e., reciprocal miscategorization of fear/surprise and anger/disgust; Gagnon et al., 2010).

Despite the rapid development of face processing abilities in preschool children, their abilities are far removed from those of adults indicating that further development and maturation of these skills must occur into middle-to-late childhood and adolescence (De Sonneville et al., 2002). Moreover, research on the development of face processing skills during this period may be particularly important since, for most children, commencing school represents a major growth in their exposure to other individuals and opportunities for social learning (Turkstra, 2000).

It appears that both facial identity (Chung and Thomson, 1995) and facial emotion (Gross and Ballif, 1991) processing skills gradually improve with increased age throughout middle childhood. Some studies have suggested a "developmental dip" - a temporary plateau or reversal in performance gains between the ages of 10-14 years (e.g., Carey et al., 1980; Flin, 1980; Soppe, 1986); however, evidence for this is inconsistent (e.g., Diamond et al., 1983; Karayanidis et al., 2009). Interpretation of the literature on both facial identity and facial emotion processing has been complicated by a variety of methodological considerations. The key complications are: (1) experiments that report recognition accuracy but not response speed may not be sensitive enough to reveal developmental differences (e.g., Chung and Thomson, 1995); (2) experiments that only involve face recognition tasks are unable to distinguish face processing development from general perceptual and cognitive development as recognition memory ability is known to improve with age (e.g., Cycowicz et al., 2001; Mondloch et al., 2006); and (3) experiments that use multiple tasks often do not match the tasks for level of difficulty (Herba et al., 2006).

De Sonneville et al. (2002) and McGivern et al. (2002) are the first studies to show the importance of measuring speed of responding when examining the development of face processing in typically developing children. These studies showed reaction-time (RT) differences reflecting a trajectory of performance improvement across age that accuracy measures alone were not able to reveal. These two studies also compared developmental patterns of processing facial and non-facial stimuli. However, since neither of the studies equated facial and non-facial tasks for difficulty, differences in developmental patterns between facial and non-facial tasks may be simply due to differences in task difficulty rather than specific developmental patterns for face processing or differential maturation of facial identity vs. facial emotion processing skills.

Indeed, facial expression processing shows substantial intrasubject and inter-task variability (Harrigan, 1984; Markham and Adams, 1992; Herba et al., 2008) as well as evidence of ceiling effects, at least for some emotions (happiness, anger, fear). This suggests that non-specific processes, such as differences in level of verbal functioning between age groups or in discrimination difficulty between stimuli or tasks, may contribute to discrepancies between studies. A number of studies have attempted to minimize such confounds by employing matching tasks that reduce the effects of memory load and differences in verbal functioning (De Sonneville et al., 2002; McGivern et al., 2002; Herba et al., 2006; Karayanidis et al., 2009). These studies show continued improvements in both facial identity and facial emotion matching throughout childhood and early adolescence. In early childhood, Karayanidis et al. (2009) reported differential preference for eye and mouth features in identity and emotion matching, respectively. This differential preference peaked at an earlier age for identity than for emotion judgments. Contrary to the findings of De Sonneville et al. (2002), this study found that children were generally slower and less accurate in making identity judgments than emotion judgments. However, again, there was no explicit attempt to ensure equivalent psychometric properties across tasks (Chapman and Chapman, 1978).

Although there are often early signs and indications, many childhood disorders of cognition are given a confirmation of diagnosis within middle-to-late childhood years (Howlin and Asgharian, 1999). Thus, developmental processes occurring during this period may be particularly important in terms of demarcating typical vs. atypical development. A better understanding of face processing development during these years is therefore especially pertinent. Bruce et al.'s (2000) battery of face processing skills for 4- to 10-year olds is successful in part because it involves multiple tasks matched for similar cognitive demands. However, all of these tasks involve elements of face processing. Arguably, a battery that is informed by performance of children of different ages at face- and nonface processing tasks of matched difficulty could have even greater diagnostic utility.

The current study aims to assess the development of facial identity and facial emotion processing across a broad age range of children and adolescents (6- to 15-years old) using tasks that are equated for discrimination difficulty in adults. Emotion and identity facial matching tasks were applied in order to minimize potential verbal and memory confounds. A butterfly wing patternmatching task was designed using stimuli adapted from Rhodes et al. (2004) to control for non-specific improvement in discrimination ability for complex visual stimuli. For each task, stimuli were carefully selected based on similarity ratings in a young adult group and tested to confirm that the three tasks were equated for discrimination difficulty. The combination of a broad age range and a matching paradigm that measures both speed and accuracy of discrimination with facial emotion, facial identity, and nonfacial identity tasks that are equated for difficulty will allow this study to address some of the gaps in the existing literature and provide a framework for the more rigorous characterization of 
face processing skills across the developmental span. This will help provide a robust baseline for the interpretation of face processing abnormalities associated with clinical conditions.

\section{MATERIALS AND METHODS PARTICIPANTS}

Ninety-two children were recruited through two local private schools. Participants were aged between 5 years, 7 months and 15 years, 7 months of age and included 49 boys and 43 girls. In order to run between group analyses comparable to those of most previous papers in the area, participants were divided into three age groups of approximately equal sample size using convenient cutpoints: younger $(5-7.9$ years; $n=29)$, middle ( $8-12.5$ years; $n=34)$, and older $(12.6-15.6$ years; $n=29)$ children. Initial analyses of IQ scores obtained using the Peabody Picture Vocabulary Test showed a significant IQ difference between age groups, largely due to IQ values above 130 in three children in the middle group. As these values were outside the range of IQ values of children in the other two age groups, we excluded these children from further analyses in order to eliminate statistically significant $(p>0.10)$ IQ differences between groups. Table 1 shows demographic information for the all groups. A group of adults $(n=24)$ were also recruited from the first year participant pool of the University of Newcastle for course credit.

\section{STIMULUS SET DEVELOPMENT}

The three tasks were equated for discrimination difficulty in three pilot studies conducted on separate groups of young adults. In the first phase, a group of 32 undergraduate students (mean 18.8 years, 7 male) were presented with a large number of pairs of photos and asked to give a similarity rating for each pair. These included pairs of butterfly wings from Rhodes et al. (2004), pairs of faces belonging to same or different people from the University of Stirling face database and pairs of faces displaying same or different emotions from Matsumoto and Ekman (1988). These ratings were used to select one set of stimuli for each task (i.e., 150 stimuli for the facial emotion task, 84 stimuli for the facial identity task, and 85 stimuli for the Lepidoptera task) such that the three tasks included items with equivalent mean similarity ratings. In the second phase of test development, these three sets of stimuli were presented to a different group of undergraduate students ( $n=30$, mean 19.1 years, 8 male) who were asked to respond whether the two items presented on each trial (i.e., the

Table 1 | Demographic variables for each of the four age groups.

\begin{tabular}{lllll}
\hline & $\begin{array}{l}\text { Young } \\
\text { children }\end{array}$ & $\begin{array}{l}\text { Middle } \\
\text { children }\end{array}$ & $\begin{array}{l}\text { Old } \\
\text { children }\end{array}$ & $\begin{array}{l}\text { Young } \\
\text { adults }\end{array}$ \\
\hline$N$ & 29 & 31 & 29 & 24 \\
Age (years) & & & & \\
Mean \pm SD & $6.7 \pm 0.65$ & $10.7 \pm 1.4$ & $13.8 \pm 0.88$ & $18.7 \pm 0.85$ \\
Range & $5.6-7.9$ & $8.2-12.5$ & $12.6-15.6$ & $17.9-21.2$ \\
Gender (M:F) & $17: 12$ & $15: 16$ & $16: 13$ & $10: 14$ \\
PPVT-IO & & & & \\
Mean \pm SD & $105 \pm 9$ & $110 \pm 11$ & $106 \pm 10$ & \\
Range & $87-125$ & $86-128$ & $88-126$ &
\end{tabular}

two butterfly wings, two emotional expressions, or two faces) were the same or different using the task instructions shown below. This second phase was intended to allow us to select a reduced set of stimuli for each task such that the average RT and error rate would be equivalent across the three stimulus sets. First, we excluded stimuli with high error rate $(>15 \%$ of respondents responding incorrectly) and trials with RT outside a 200- to 5000-ms window. Then we sequentially removed stimuli from each of the three sets so as to arrive at three stimulus sets, one for each task, that had the same number of stimuli $(n=32)$, had $50 \%$ same and $50 \%$ different stimuli and did not differ in mean RT and error rate for this group of participants. In the third phase of task development, we confirm that the reduced stimulus sets were equated for task difficulty in a new group of undergraduate students $(n=30$, mean 19.6 years, 13 male). This sample showed no significant difference between the three tasks in either mean RT or $d^{\prime}$. Equivalence of task difficulty across the three tasks has since been confirmed in two published studies (Enticott et al., 2008; Johnston et al., 2010) that have used these tasks in healthy adult participants.

\section{STIMULI AND TASKS}

Stimuli were presented on a Toshiba Tecra 800012.1 inch screen laptop computer using SuperLab software. Each task (Butterfly, Identity, and Emotion) was presented in one block of 32 stimuli. Each stimulus consisted of a pair of items that were either the "same" or "different." Stimuli were presented for a maximum of $7 \mathrm{~s}$ or until a response was emitted. Participants were instructed to make a "same" or "different" response using the response box positioned on a table in front of them (Cedrus SuperLab Pro respond pad, model RB-730). For each task, stimuli were presented in a different randomized order for each participant. Task order and response (same vs. different) to hand (left vs. right) mapping were counterbalanced across participants. Response mapping remained consistent across tasks for the same participant. No response accuracy feedback was given.

\section{Lepidoptera discrimination task (butterfly)}

The Lepidoptera task was developed using photographs of butterflies and moths from Rhodes et al. (2004). These photos were edited using Photoshop so that only one wing was displayed in each photograph. The final set of stimuli included in this task consisted of 16 pairings of same and 16 pairings of different Lepidoptera wings presented in either landscape or portrait position (i.e., 22 portrait pairs, 3 landscape pairs, and 7 portrait-landscape pairs). Participants were required to respond to the question "Do these wings belong to the same butterfly?"

\section{Facial identity discrimination task (identity)}

The final stimulus set for this task included 32 faces of Caucasian actors (19 females) depicting a neutral facial expression from the University of Stirling face database (http://pics.stir.ac.uk/cgi-bin/ PICS/New/pics.cgi). The battery included three exemplars of the same person including two different full frontal views and one 3/4 frontal view. As the full frontal views of the same person tended to be very easily identified as "same," we also included pairings of 4/4 and 3/4 pictures for both same and different face pairings. It was thought that this would require some level of mental 
manipulation, broadly equivalent to that required for mentally rotating left and right or landscape and portrait butterfly wings or comparing facial expressions across different people. Stimuli were $12.5 \mathrm{~cm} \times 16.5 \mathrm{~cm}$ black and white images shown against a black background. The 32 stimuli included 14 pairs of full frontal views, 15 pairs of full and 3/4 frontal views, and 3 pairs of 3/4 frontals views. The set comprised of 16 pairs of same and 16 pairs of different individuals paired for gender. Same pairings included different views of the same person. Different pairings were selected based on similarity ratings from the pilot studies and so that the two faces were matched for age, gender, and hairstyle. Participants were asked to respond to the question "Are these two photos of the same person?" (Figure 1).

\section{Facial affect discrimination task (emotion)}

Thirty-two faces were selected from Matsumoto and Ekman (1988) JACFEE/JACNeuF stimulus sets. The original stimuli were reduced to $8.5 \mathrm{~cm} \times 6 \mathrm{~cm}$ images and converted to grayscale to reduce color cues and increase consistency across tasks. The task included 16 pairs of same and 16 pairs of different facial emotional expressions. These consisted of adult male and female faces expressing a positive (happy or surprised), negative (sad or angry), or a neutral expression. These 32 pairs included 10 male face pairs, 10 female face pairs, and 12 male-female face pairs, with roughly equal representation of the five emotions (i.e., four happy and three of all other emotions. For "different" pairings, each emotion expression was presented

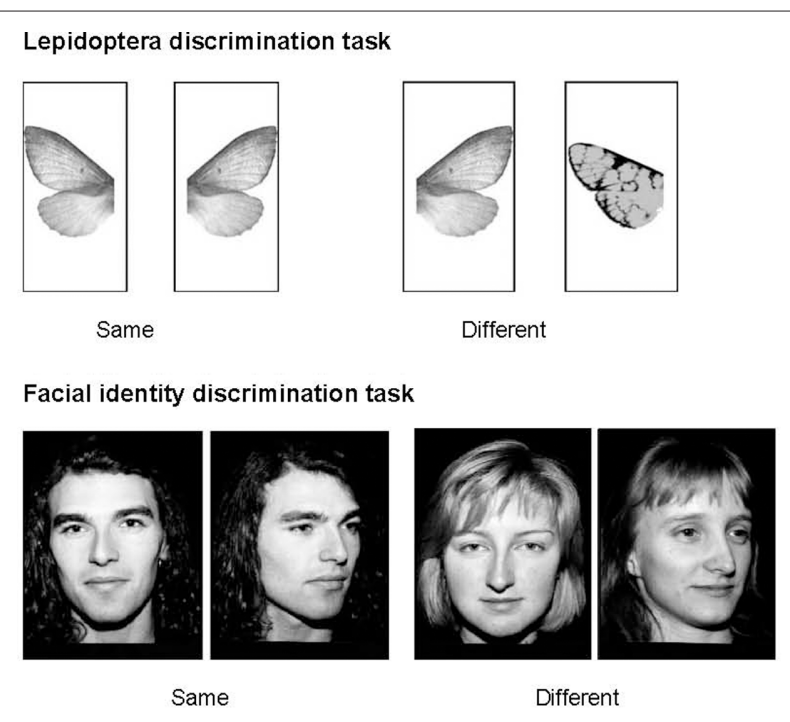

Facial emotion discrimination task

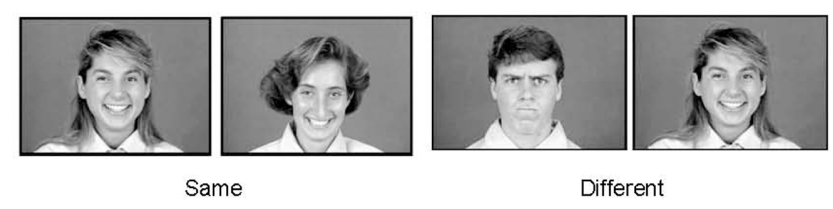

FIGURE 1 | Examples of same and different stimulus pairs from each of the three tasks. on seven stimuli, except for neutral which was presented on four stimuli. Participants were asked to respond to the question "Do these two people feel the same way?" (Figure 1).

\section{PROCEDURE}

Written instructions were presented on the screen before the commencement of each task and were read to the participant by the researcher. Each task was preceded by six practice trials. In the first two practice trials, participants were presented with a stimulus and the correct discrimination response (e.g., "these two photographs are the same"). For the four remaining practice trials, participants were encouraged to examine the photographs to determine if they were the "same" or "different," and to then press the appropriate button. Participants received immediate accuracy feedback (the word "correct" or "incorrect" appeared on the monitor) and, if incorrect, were given the opportunity to study the slide again. Upon completion of practice trials, each task was presented in a continuous block and the participant was instructed to classify each stimulus ("same" or "different") as quickly and as accurately as possible. The experimental session lasted approximately $30 \mathrm{~min}$, including rest intervals.

\section{DATA ANALYSIS}

Responses were coded as correct if the appropriate key was pressed at least $250 \mathrm{~ms}$ after and within $7 \mathrm{~s}$ of stimulus onset. Late and incorrect responses were recorded, but only correct responses were used to estimate mean RT. The dependent variables were mean RT for correct responses, and the signal detection measures of hit rate (HR), false alarm (FA) rate, discriminability $\left(d^{\prime}\right)$, bias $(\beta)$, and proportional bias $\left(\beta_{\mathrm{p}}\right)$. A hit was defined as a correctly identified same pair (Green and Swets, 1966). A FA was defined as an incorrectly identified different pair (e.g., responding same to a different trial). HR and FA rate were used to calculate the signal detection parameters, including sensitivity ( $d^{\prime}$ : discriminability) and criterion ( $\beta$ : bias). $d^{\prime}$ is a sensitivity measure and, in the present study, provides a measure of how well a subject discriminated "same" stimuli (increasing $d^{\prime}$ indicates higher sensitivity (Green and Swets, 1966). $\beta$ produces a measure of how certain (based on personal factors, previous experience) a participant needs to feel before they will judge whether the stimulus is "same" or "different" and provides a measure of bias toward one type of responding (Green and Swets, 1966). As the interpretation of $\beta$ depends on the level of $d^{\prime}$, we analyzed proportional beta $\left(\beta_{\mathrm{p}}\right)$ estimated as $\beta_{\mathrm{p}}=\beta / d^{\prime}$. Thus, $\beta_{\mathrm{p}}=0.5$ means unbiased responding for any sensitivity level, while $\beta_{\mathrm{p}}<0.5$ indicates a bias toward "same" responses and $\beta_{\mathrm{p}}>0.5$ indicates a bias toward "different" responses.

\section{Age group analyses}

The first phase of analyses was run using discrete age groups in order to be readily comparable with most previous literature in the area which uses non-continuous age groups. The three tasks were compared on each dependent variable with age group and gender as between subject variables 4 Age Group $\times 2$ Gender $\times 3$ Task (Butterfly, Identity, Emotion) mixed design ANOVA, with Greenhouse-Geisser correction on the task factor. Significant effects of task were examined using two planned contrasts with Bonferroni adjustment of family wise error rate $(a=0.05 / 2=0.025)$. One compared discrimination of physical features of non-facial 
(Butterfly) vs. facial (Identity) stimuli and the other compared discrimination of faces on the basis of physical features (Identity) vs. facial expression (Emotion). Significant effects of age were examined in the first instance using repeated contrasts between successive age groups. This consistently resulted in no difference between middle and older children groups (see also Figure 2). Therefore, in order to maximize statistical power when examining developmental changes, we compared young children and adults to the combined group of middle and older children in two separate ANOVAs (2 Age Group $\times 2$ Gender $\times 3$ Task mixed design) using Bonferroni correction on family wise error rate $(a=0.05 / 2=0.025)$ for the age factor. So, when comparing young and older children or older children and adults, age main effects, and interactions are only reported if the $F$-value is significant at $p<0.025$. Independent group $t$-tests were used to compare groups for each task alone $(a=0.05 / 3=0.016)$.

\section{Analyses with age as continuous factor}

The second phase of analyses used age as a continuous variable. Firstly we ran correlations between each variable for each task and age. Secondly in order to examine whether the slope of the age effect on each variable differs across the three tasks we re-ran the above ANOVAs using age as a covariate instead of a group factor. Specifically, linear and quadratic age trends were examined by

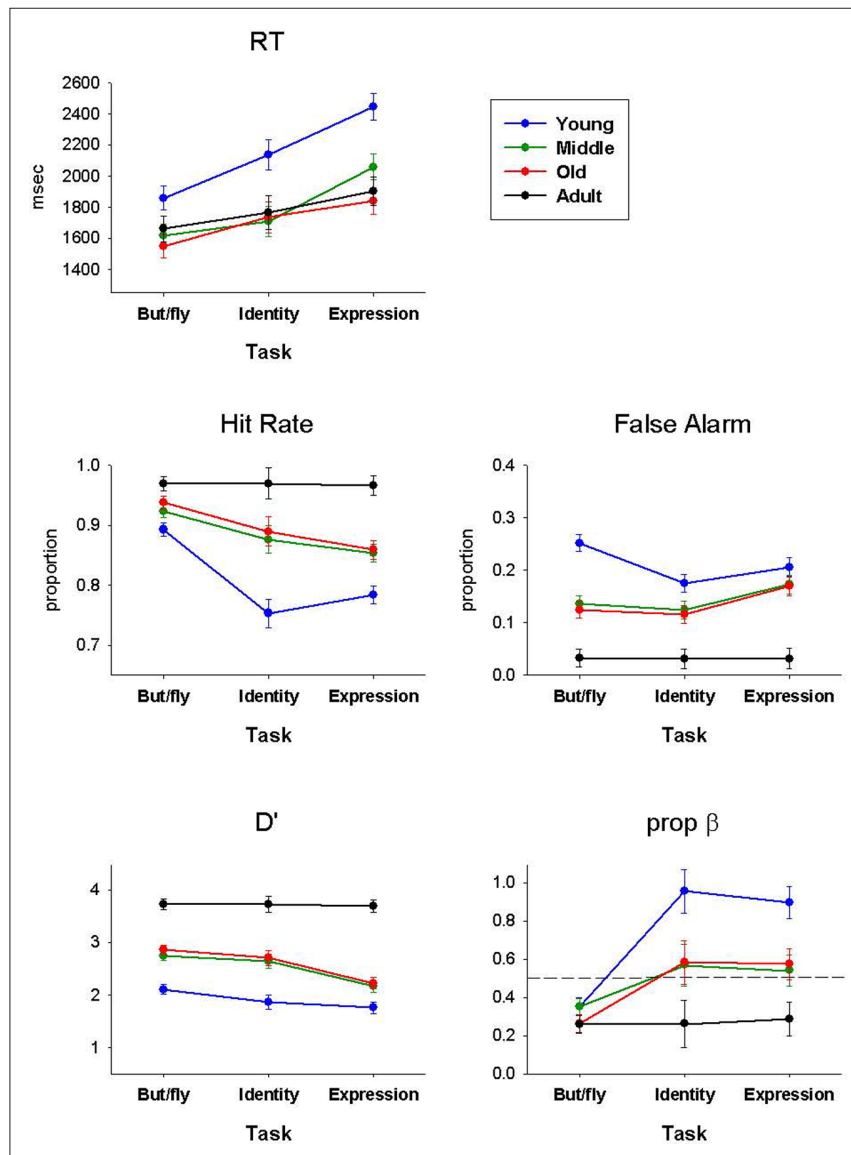

FIGURE 2 | Behavioral measures for each task arranged by age group. including centered age and centered age squared as covariates in analyses of each dependent variables using a one ANOVA on Task (3) with age and age $\mathrm{e}^{2}$ as covariates.

\section{RESULTS}

\section{OVERALL GENDER EFFECTS}

There were widespread gender differences across the age range. Specifically, females responded more quickly than males [1750 vs. $1970 \mathrm{~ms}, F(1,106)=9.6, p=0.002$ ], had significantly higher HR $[0.90$ vs. $0.88, F(1,106)=4.3, p=0.041]$ and marginally lower FA rate $[0.12$ vs. $0.14, F(1,106)=3.1, p=0.077]$. Females also showed greater $d^{\prime}[2.8$ vs. 2.6, $F(1,106)=5.1, p=0.026]$ than males, but there was no gender difference in $\beta_{\mathrm{p}}$. Despite this overall better performance for females as compared to males, there was no interaction between gender and either age group or task. Therefore, the data in Figure 2 are shown averaged over gender.

\section{AGE AND TASK EFFECTS}

The overall analysis comparing the four age groups resulted in widespread main effects of age group [RT: $F(3,110)=7.3, p<0.001$; HR: $F(3,110)=30.5, p<0.001$; FA: $F(3,110)=36.5, p<0.001 ; d^{\prime}$ : $F(3,110)=73.6, p<0.001]$ and task $[\mathrm{RT}: F(2,220)=40.12, p<0.001$; HR: $F(2,220)=18.4, p<0.001$; FA: $F(2,220)=5.25, p=0.006 ; d^{\prime}:$ $\left.F(2,220)=15.8, p<0.001 ; \beta_{\mathrm{p}}: F(2,220)=8.5, p<0.001\right]$, as well as age group by task interactions [HR: $F(6,220)=3.5, p=0.004$; FA: $F(6,220)=2.6, p=0.02 ; d^{\prime}: F(6,220)=2.2, p=0.05 ; \beta_{\mathrm{p}}$ : $F(6,220)=2.5, p=0.02]$. As shown in Figure 2, the effects of task differed substantially in young children compared to older children, as well as in older children compared to adults. As noted above (Data Analyses), the middle and older children showed very similar performance outcomes and statistical analyses comparing these two groups directly revealed no significant group or group by task effect on any of the performance measures (all $p>0.05$ ), and therefore subsequent analyses averaged across these two groups. In the following sections, we first report the effects of task in the Adult group, and then examine differences between young (5-8 years) and the combined group of 8- to 15-year-old children as well as between the latter and adults.

\section{TASK EFFECTS IN ADULTS}

While there was a substantial change in RT across the three tasks, increasing from $1660 \mathrm{~ms}$ for the Butterfly task to $1760 \mathrm{~ms}$ for the Identity task and 1900 ms for the Expression task, the main effect of task on RT was only marginally significant $[F(2,48)=2.9$, $p=0.067$. As shown in Figure 2, adults showed little difference between tasks on measures of accuracy and discriminability. A significant main effect of task on $\operatorname{HR}[F(2,48)=7.5, p=0.008]$ reflected a reduction in $\mathrm{HR}$ of just $1 \%$ for the Expression as compared to the Identity task [ 96 vs. $97 \% ; F(1,24)=7.1, p=0.014$ ] and is likely to have arisen because of reduced measurement variability due to ceiling effects. Similarly, a task main effect on $\beta_{\mathrm{p}}$ $[F(2,48)=6.8, p=0.007]$ reflected very small increase in bias toward a same response for the identity as compared to the expression task $[F(1,24)=7.0, p=0.014]$. So, globally the three tasks produced similar performance in young adults, with small but significant trends suggesting that the facial expression discrimination task was somewhat more difficult. 


\section{MIDDLE AND OLD CHILDREN VS. ADULTS'}

Although children responded as fast as adults ( $p>0.5$ ), they had lower HR [ 89 vs. $97 \% ; F(1,83)=40.7, p<0.001]$ and higher FA rate $[14$ vs. $3 \% ; F(1,83)=61.6, p<0.001]$ than adults. So, children tended to respond as fast as but less accurately than adults, being more likely to respond "different" to same items and "same" to different items. This is reflected in lower $d^{\prime}[F(1,83)=136.1, p<0.001]$ and higher $\beta_{\mathrm{p}}[F(1,83)=13.4, p<0.001]$ than adults reflecting a shift toward less bias, especially for the face discrimination tasks, as discussed below.

A significant task $\times$ age group interaction was obtained for $d^{\prime}[F(2,166)=5.8, p=0.006]$, with marginal effects on HR $[F(2,166)=4.1, p=0.03]$ and FA rate $[F(2,166)=2.6, p=0.074]$. The children had a higher HR on the butterfly as compared to the identity task $[F(1,59)=8.2, p=0.006]$, and a higher FA rate on the expression as compared to the identity task $[F(1,59)=11.2$, $p=0.001]$. Signal detection measures showed a lower $d^{\prime}$ for the expression task as compared to the identity task $[F(1,59)=13.2$, $p=0.001]$, as well as a higher $\beta_{\mathrm{p}}$ on the identity as compared to the butterfly task $[F(1,59)=9.2, p=0.004]$. Direct comparisons between the combined child and adult groups on each task separately revealed that, on the butterfly task, children had significantly lower HR, higher FA rate, and lower $d^{\prime}$ than adults $[t(83)>5.9$, $p<0.001]$, whereas all performance measures except RT were significantly different between groups for both facial identity and expression tasks $[t(83)=3.5-16.7, p<0.001]$. Gender did not significantly interact with either task or age group $(p>0.06)$.

In summary, despite being as fast as adults, children aged 8-15 years performed less accurately and had lower sensitivity than adults, across all three tasks. In addition, for both face discrimination tasks, these children showed an elimination of the "same" bias shown by adults. This suggests age-related differences in speed/accuracy trade-off, with children showing less accurate responding in return for equal speed, especially on the facial discrimination tasks. Most importantly, children showed poorer sensitivity on the expression discrimination task than the identity discrimination task, suggesting greater difficulty in discriminating faces on the basis of expression than identity.

\section{YOUNG VS. MIDDLE AND OLD CHILDREN}

As shown in Figure 2, younger children (5.6-7.9 years) performed all tasks more poorly than the middle and old children groups. Young children were almost $400 \mathrm{~ms}$ slower than older children [2146 vs. $1752 \mathrm{~ms} ; F(1,87)=17.3, p<0.001$ ]. While young children were significantly slower than other children on each of the three tasks $[t(87)>3.1, p<0.003]$, they were significantly slower on the identity task as compared to the butterfly task $[F(1,28)=14.5$, $p<0.001]$ and on the expression task as compared to the identity task $[F(1,28)=12.8, p<0.001]$.

Young children also had a lower HR [ 81 vs. $89 \% ; F(1,87)=26.3$, $p<0.001$ ] and higher FA rate [21 vs. $14 \% ; F(1,87)=19.2, p<0.001$ ] than older children. The effect of age on HR was significant for both identity and emotion tasks $[t(87)>46.42, p<0.001]$, whereas the effect on FA rate was significant for butterfly task only $[t(87)>49.36$,

${ }^{1}$ Note that we also ran the analysis comparing the old children and adult groups directly and derived the same pattern of statistical results. Similarly, comparison between young and middle children groups resulted in similar results as the analysis reported below between young and the combined middle/old group, but with smaller statistical significance values. $p<0.001]$. Task comparisons within the young group showed lower $\mathrm{HR}$ as well as lower FA rate for the identity as compared to the butterfly task $[F(1,28)=18.5, p<0.001 ; F(1,28)=1.6, p<0.001$, respectively], but no difference between identity and expression tasks. These results suggest that younger children were more likely to respond "different" to facial stimuli in both identity and expression tasks, as compared to the butterfly task. Signal detection measures showed an overall reduction in $d^{\prime}$ and increase in $\beta_{\mathrm{p}}$ in young as compared to older children $[F(1,87)=31.7, p<0.001$; $F(1,87)=12.7, p<0.001$, respectively]. The young group showed a significant increase in $\beta_{\mathrm{p}}$ for the identity as compared to the butterfly task $[F(1,28)=17.3, p<0.001]$. So, while young and older children did not differ in bias on the butterfly task, young children showed a larger bias toward a "different" response on both tasks requiring discrimination of faces. Gender did not significantly interact with either task or age group ( $p>0.08)$.

In summary, young children found all tasks harder than older children, with slower responding, lower HR, and higher FA date, resulting in overall reduced sensitivity. More importantly, younger children seemed to find the two facial discrimination tasks more challenging than the non-facial (butterfly) discrimination task, as suggested by relative increase in $\mathrm{RT}$, reduction in $\mathrm{HR}$, and increase in response bias for the facial tasks. Note, however, that other than an increase in RT, there was no evidence that the younger children found the facial expression more challenging than the facial identity discrimination task.

\section{AGE COVARIATE ANALYSES}

Pearson correlation coefficients showed a significant negative correlation between age and RT for the facial expression discrimination task $(r=-0.388, p<0.001,2$-tailed $)$ with similar but non-significant trends for the other two tasks. Increasing age was associated with higher HR $(r>0.445, p<0.001)$ and lower FA rate $(r<-0.487$, $p<0.001)$ on all tasks. This was reflected in a significant age-related increase in $d^{\prime}$ for all tasks $(r>0.740, p<0.001)$ and reduction in $\beta_{\mathrm{p}}$ for the two facial discrimination tasks $(r<-0.393, p<0.001)$.

As expected from the correlational analyses, age was a significant linear covariate on each of the dependent variables $[F(1,110)>16.7$, $p<0.001]$. In addition, the quadratic trend of age was significant for RT alone $[F(1,110)>10.7, p<0.001]$ indicating a sharp decline in $\mathrm{RT}$ in the younger age range (Figure 3). More importantly, the slope of the linear age effect differed between tasks on RT, HR, and $\beta_{\mathrm{p}}[F(2,220)>5.20$, $p<0.009$ ] indicating a sharper linear age slope for the facial identity than the butterfly task, but no difference between the two facial tasks. Notably, for $d^{\prime}$ there was a significant quadratic modulation of task by age $[F(2,220)=5.29, p<0.007]$. As shown in Figure 3 (middle), while $d$ improved linearly with age for both butterfly and facial identity tasks, it showed a curvilinear effect in facial expression task, with little improvement over 5-10 years followed by some improvement over 10-15 years and a sharp rise in young adults.

\section{DISCUSSION}

The primary purpose of this work was to explore the differential development of face processing skills in middle-to-late childhood. Unlike most prior work, our paradigm was designed to minimize two commonly occurring confounds which can reduce sensitivity to age-related effects, i.e., task difficulty and general developmental improvement in perceptual and cognitive processing unrelated to 

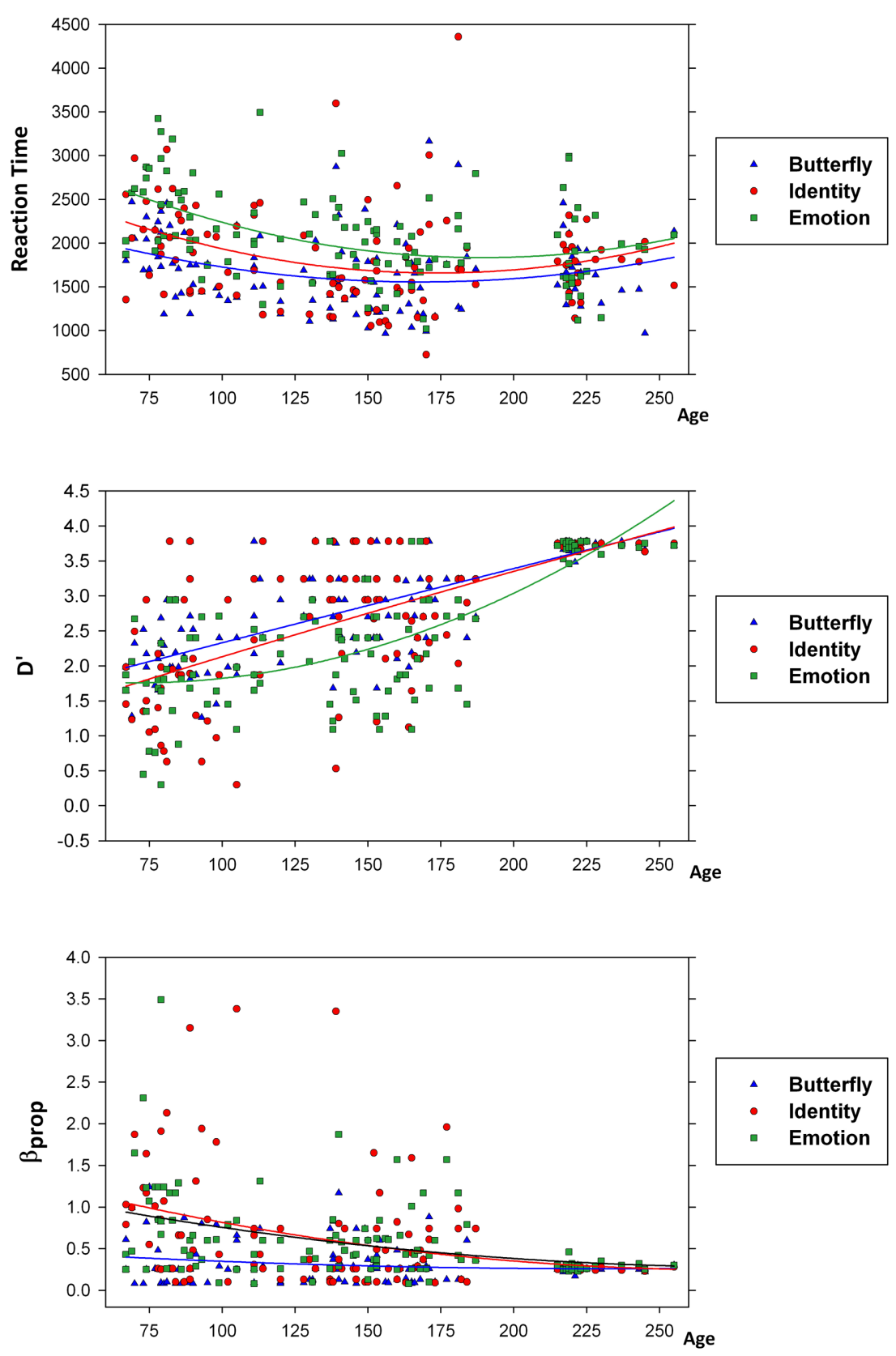

FIGURE 3 | Scatterplots of reaction time, $d^{\prime}$ and $\beta_{p}$ against age (in months) with quadratic lines of best fit.

face processing. We controlled for task difficulty by carefully matching tasks for discrimination difficulty in adults and we controlled for general developmental improvements by including a non-facial discrimination task of equivalent difficulty.

The main finding of this work is that in 8- to 15 -year olds the development of facial emotion discrimination ability lags behind that of facial identity discrimination. As shown in Figures 2 and 3, older children were significantly less accurate at the expression discrimination task than they were at the identity and butterfly discrimination tasks. This was reflected in $d^{\prime}$ scores as well as HR and FAs (RTs were also greater for the emotional expression task, but this did not reach statistical significance). In contrast, neither the adult nor young child group displayed any such differential performance for facial expression discrimination in comparison to the other two tasks. The fact that our tasks were equated for discrimination difficulty in adults is critical in interpreting this effect. This allows us to conclude that the differences between older children and adults reflect a real difference in underlying processes rather than simply a difference in task difficulty. In other words, the observed differences can not be attributed to the underlying features of one set of stimuli (i.e., emotional face set) being inherently harder to discriminate than the other set of stimuli (i.e., identity face set). 
Rather our findings strongly indicate that, in older children, the neural/psychological processes involved in expression discrimination lag behind those of identity discrimination.

Our findings are particularly interesting when set against the broader context of earlier research. In agreement with our findings, De Sonneville et al. (2002) found that adolescent children were generally better (faster and more accurate) at matching for facial identity than at matching for emotional expressions. In contrast, Karayanidis et al. (2009) reported that adolescents were better at facial emotional expression matching than similar identity judgments. This discrepancy could have resulted from differences in discrimination difficulty among the stimulus sets for expression and identity matching used in these two studies. As the current study controlled for discrimination difficulty in adults, we can more strongly conclude that for the 8- to 15-year-old children, facial expressions are more challenging to discriminate than facial identity.

That processing of emotional expression lags behind that of identity is not entirely surprising. Recent work by Tonks et al. (2007) found that young people at around the end of the preteen stage show marked improvement at recognizing emotion from one's eyes alone. Interestingly, children in the older age group were significantly faster at making expression judgments than children in the middle age group. It could be the case that part of what develops here is that the older participants need not scan the entire face to make their judgment, responding more quickly based on the eyes alone, whereas the younger participants are compelled to scan more of the picture before making a response. Interestingly, Karayanidis et al. (2009) showed that younger children attend more to the mouth region than the eye region when making emotion judgments (and vice versa for identity judgments). Hence, what develops in later childhood may include a fuller understanding of the subtle emotional cues that can be read from the eyes. This notion is consistent with the finding that younger children have problems in identifying false smiles or masking smiles than either older children or adults (Gosselin et al., 2002): genuine smiles are more likely to be "Duchenne Smiles" which involve high information content in the eye region. Future longitudinal studies involving eye-tracking could help validate this hypothesis. Additionally, it has been argued that around the preteen years, the importance of social interaction among peers rises dramatically (Turkstra, 2000). By this reasoning, at this key period of adolescence, young people may become much more proficient at gleaning emotional expression cues from faces. Consequently, children at this age would become more practiced and show greater proficiency at facial recognition tasks.

It is interesting to note that expression processing performance did not seem to compare adversely to identity processing performance for the youngest participants. This could be explained in one of two ways. The first, less interesting, possibility is that the youngest children were subject to a floor effect. However, this is unlikely as even the youngest age group were performing well above chance. The second, more interesting, possibility is that the older children's performance on the expression tasks suffers because they are engaged in a period of representational redescription (KarmiloffSmith, 1994). By this account, adolescents are beginning to process expression in new ways (e.g., by putting more focus on the eyes alone) but are not yet entirely proficient at this. Thus, their performance suffers until they are more practiced whereupon there is a significant boost in their ability to process emotional expressions.
The results of the butterfly task are also worth noting. As expected, there was a clear developmental effect in speed and accuracy of responding with the older participants outperforming the younger participants. Such findings fit with those of De Sonneville et al. (2002), which also found significant improvement on nonfacial matching tasks. More interesting, however, is the finding that the youngest participants were significantly faster at the butterfly task than they were at either of the face processing tasks despite the fact their sensitivity across the tasks was generally level. This finding is counterintuitive if one assumes that our brains are intrinsically wired to efficiently process faces (Bruce and Young, 1986). Such efficiencies should translate to faster processing of face stimuli than non-face stimuli given equal levels of accuracy - the opposite of the observed effect.

Nonetheless, that fact that there was a difference (albeit in the unexpected direction) in the RTs in the face vs. non-face matching task is indicative of distinct face processing mechanisms in the younger children. One possible explanation for young children's apparent superiority at the non-face matching task is that the preadolescent neural pathways involved in face processing are not fully developed in comparison with those subserving non-face identity matching tasks. A recent MEG study with children of similar ages (Kylliainen et al., 2006) provides some evidence in favor of this possibility. In this study, face stimuli seen by adults resulted in very short latency activity in the right inferior occipito-temporal pathways. However, in children, this activity was significantly less prominent and not lateralized.

Finally some discussion of the butterfly task in comparison to the face identity task is warranted. Sensitivity scores (i.e., $d^{\prime}$ ) would seem to indicate that identity processing performance at all ages can be predicted by general cognitive performance. Indeed, the purpose of the butterfly task was to ascertain the degree to which any age-related improvement in performance of face processing tasks was the result of improvement in general cognitive ability. An examination of the bias scores $\left(\beta_{\mathrm{p}}\right)$ however, reveals a very different developmental pattern for the butterfly task and the face identity task. Specifically, participants of all ages had a significant bias toward responding "different" in the butterfly task. In contrast, there were strong age differences in bias for facial stimuli: while the youngest participants showed a strong "same" bias, adults had a significant "different" bias, and remaining children showed no bias. The current data do not provide a clear explanation for this developmental trend. However, it strongly suggests that young children process faces and butterflies very differently, whereas this difference is gradually eliminated in older children and adults. While there is a growing literature on how the adult brain processes face vs. non-face stimuli (e.g., Kanwisher and Yovel, 2005; Mercure et al., 2008), future electrophysiological, and neuroimaging investigations with children will help explain how such stimuli are differentially processed in the developing brain.

\section{ACKNOWLEDGMENTS}

The authors would like to note Alicia Sercombe's invaluable contribution to stimulus production, prepiloting, and selection and to thank Rebecca Smith for her help with data collection. Patrick J. Johnston was supported by an NHMRC Clinical Training Fellowship. The project was supported by the Hunter Medical Research Institute and the University of Newcastle. 


\section{REFERENCES}

Boyatzis, C. J., Chazan, E., and Ting, C.Z. (1993). Preschool children's decoding of facial emotions. J. Genet. Psychol. 154, 375-382.

Bruce, V., Campbell, R. N., DohertySneddon, G., Import, A., Langton, S., McAuley, S., and Wright, R. (2000). Testing face processing skills in children. Br. J. Dev. Psychol. 18, 319-333.

Bruce, V., and Young, A. (1986). Understanding face recognition. $\mathrm{Br}$. J. Psychol. 77, 305-327.

Bushnell, I., Sai, F., and Mullen, J. (1989). Neonatal recognition of the mother's face. Br. J. Dev. Psychol. 7, 3-15.

Carey, S., Diamond, R., and Woods, B. (1980). Development of face recognition: a maturational component? Dev. Psychol. 16, 257-269.

Chapman, L. J., and Chapman, J.P. (1978). The measurement of differential deficit. J. Psychiatr. Res. 14, 303-311.

Chung, M.S., and Thomson, D.M. (1995). Development of face recognition. $\mathrm{Br}$. J. Psychol. 86, 55-87.

Cycowicz, Y.M., Friedman, D., Snodgrass, J.G., and Duff,M. (2001). Recognition and source memory for pictures in children and adults. Neuropsychologia 39, 255-267.

de Haan, M., and Nelson, C. A. (1997). Recognition of the mother's face by six-month- old infants: a neurobehavioral study. Child Dev. 68, 187-210.

De Sonneville, L. M., Verschoor, C. A., Njiokiktjien, C., Op het Veld, V., Toorenaar, N., and Vranken, M. (2002). Facial identity and facial emotions: speed, accuracy, and processing strategies in children and adults. J. Clin. Exp. Neuropsychol. 24, 200-213.

Diamond, R., Carey, S., and Back, K. J. (1983). Genetic influences on the development of spatial skills during early adolescence. Cognition 13, 167-185.

Enticott, P.G., Johnston, P. J., Herring, S.E., Hoy, K. E., and Fitzgerald, P. B. (2008). Mirror neuron activation is associated with facial emotion processing. Neuropsychologia 46, 2851-2854.

Flin, R. H. (1980). Age effects in children's memory for unfamiliar faces. Dev. Psychol. 16, 373-374.

Gagnon, M., Gosselin, P., Hudon-ven der Buhs, I., Larocque, K., and Milliard, K. (2010). Children's recognition and discrimination of fear and disgust facial expressions. J. Nonverbal Behav. $34,27-42$
Gao, X., and Maurer, D. (2009). Influence of intensity on children's sensitivity to happy, sad, and fearful facial expressions. J. Exp. Child. Psychol. 102 503-521.

Gosselin, P., Beaupré, M., and Boissonneault, A. (2002). Perception of genuine and masking smiles in children and adults: sensitivity to traces of anger. J. Genet. Psychol. 163, 58-71.

Green, D. M., and Swets, J.A. (1966). Signal Detection Theory and Psychophysics. New York: Wiley.

Gross,A.L., and Ballif, B. (1991). Children's understanding of emotion from facial expressions and situations: a review. Dev. Rev. 11, 368-398.

Harrigan, J. A. (1984). The effects of task order on children's identification of facial expressions. Motiv. Emot. 8, 157-169.

Haxby, J.V., Hoffman, E. A., and Gobbini, M. I. (2000). The distributed human neural system for face perception. Trends Cogn. Sci. 4, 223-232.

Herba, C. M., Benson, P., Landau, S. Russell, T., Goodwin, C., Lemche, E. Santosh, P., and Phillips, M. (2008). Impact of familiarity upon children's developing facial expression recognition. J. Child Psychol. Psychiatry 49, 201-210.

Herba, C. M., Landau, S., Russell, T. Ecker, C., and Phillips, M. L. (2006). The development of emotion-processing in children: effects of age, emotion, and intensity. J. Child Psychol. Psychiatry 47, 1098-1106.

Howlin, P., and Asgharian, A. (1999). The diagnosis of autism and asperger syndrome: findings from a survey of 770 families. Dev. Med. Child Neurol. 41, 834-839.

Johnson, M. H., Dziurawiec, S., Ellis, H., and Morton, J. (1991). Newborns' preferential tracking of face-like stimuli and its subsequent decline. Cognition 40, 1-19.

Johnston, P. J., Devir, H., and Karayanidis, F. (2006). Facial emotion processing in schizophrenia: no evidence for a deficit specific to negative emotions in a differential deficit design. Psychiatry Res 143, 51-61.

Johnston, P. J., Enticott, P. G., Mayes, A. K., Hoy, K. E., Herring, S. E., and Fitzgerald, P. B. (2010). Symptom correlates of static and dynamic facial affect processing in schizophrenia: evidence of a double dissociation? Schizophr. Bull. 36, 680-687.

Johnston, P. J., Katsikitis, M., and Carr V. J. (2001). A generalised deficit can account for problems in facial emotion recognition in schizophrenia. Biol. Psychol. 58, 203-227.

Johnston, P. J., McCabe, K., and Schall, U. (2003). Differential susceptibility to performance degradation across categories of facial. Biol. Psychol. 63 , 45-58.

Kanwisher, N., and Yovel, G. (2005). The fusiform face area: a cortical region specialized for the perception of faces. Philos. Trans. R. Soc. Lond., B, Biol. Sci. 361, 2109-2128.

Karayanidis, F., Kelly, M., Chapman, P., Mayes, A., and Johnston, P. (2009). Facial identity and facial expression matching in 5-12-year-old children and adults. Infant Child Dev. 18 404-421.

Karmiloff-Smith, B. A. (1994). Beyond modularity: a developmental perspective on cognitive science. Int. J. Lang. Commun. Disord. 29, 95-105.

Kylliainen, A., Braeutigam, S., Hietanen, J. K., Swithenby, S. J., and Bailey, A. J. (2006). Face-and gaze-sensitive neural responses in children with autism: a magnetoencephalographic study. Eur. J. Neurosci. 24, 2679.

Leppänen, J. M., and Nelson, C.A. (2006) The development and neural bases of facial emotion recognition. Adv. Child. Dev. Behav. 34, 207-246.

Markham, R., and Adams, K. (1992). The effect of type of task on children's identification of facial expressions. $J$. Nonverbal Behav. 16, 21-39.

Matsumoto, D., and Ekman, P. (1988). Japanese and Caucasian Facial Expressions of Emotion (JACFEE) [Slides]. San Francisco, CA: Intercultural and Emotion Research Laboratory, Department of Psychology, San Francisco State University.

McGivern, R. F., Andersen, J., Byrd, D. Mutter, K. L., and Reilly, J. (2002). Cognitive efficiency on a match to sample task decreases at the onset of puberty in children. Brain Cogn. 50, 73-89.

Mercure, E., Dick, F., Halit, H., Kaufman, J., and Johnson, M. H. (2008) Differential lateralization for words and faces: category or psychophysics. J. Cogn. Neurosci. 20, 2070-2087.

Mondloch, C. J., Maurer, D., and Ahola, S. (2006). Becoming a face expert. Psychol. Sci. 17, 930.

Pascalis, O., de Schonen, S., Morton, J. Deruelle, C., and Fabre-Grenet, M. (1995). Mother's face recognition in neonates: a replication and an extension. Infant Behav. Dev. 18, 79-85.

Phillipot, P., and Feldman, R.S. (1990).Age and social competence in preschooler's decoding of facial expression. Br. J. Soc. Psychol. 29, 43-54.

Rhodes, G., Byatt, G., Michie, P. T., and Puce, A. (2004). Is the fusiform face area specialized for faces, individuation, or expert individuation? J. Cogn. Neurosci. 16, 189-203.

Smith, M., and Walden, T. (1998). Developmental trends in emotion understanding among a diverse sample of African-American preschool children. J. Appl. Dev. Psychol. 19, 177-197.

Soppe, H. J. G. (1986). Children's recognition of unfamiliar faces: developments and determinants. Int. J. Behav. Dev. $9,219$.

Southgate, V., Csibra, G., Kaufman, J., and Johnson, M. H. (2008). Distinct processing of objects and faces in the infant brain. J. Cogn. Neurosci. 20, 741-749.

Tonks, J., Williams, W. H., Frampton, I., Yates, P., and Slater, A. (2007). The neurological bases of emotional dysregulation arising from brain injury in childhood: a "when and where" heuristic. Brain Impair. 8, 143-153.

Turkstra, L. S. (2000). Should my shirt be tucked in or left out? The communication context of adolescence. Aphasiology 14, 349-364.

Walker-Andrews, A. S. (1997). Infants' perception of expressive behaviors: differentiation of multimodal information. Psychol. Bull. 121, 437-456.

Conflict of Interest Statement: The authors declare that the research was conducted in the absence of any commercial or financial relationships that could be construed as a potential conflict of interest.

Received: 03 August 2010; accepted: 07 February 2011; published online: 22 February 2011.

Citation: Johnston PJ, Kaufman J, Bajic J, Sercombe A, Michie PT and Karayanidis F (2011) Facial emotion and identity processing development in 5- to 15-year-old children. Front. Psychology 2:26. doi: 10.3389/ fpsyg.2011.00026

This article was submitted to Frontiers in Developmental Psychology, a specialty of Frontiers in Psychology.

Copyright (c) 2011 Johnston, Kaufman Bajic, Sercombe, Michie and Karayanidis. This is an open-access article subject to an exclusive license agreement between the authors and Frontiers Media SA, which permits unrestricted use, distribution, and reproduction in any medium, provided the original authors and source are credited. 